\title{
DETECTION, MODELING AND CLASSIFICATION OF MOLDINGS FOR AUTOMATED REVERSE ENGINEERING OF BUILDINGS FROM 3D DATA
}

\author{
$\underline{\text { Enrique Valero }}^{1 *}$, Antonio Adan ${ }^{2}$, Daniel Huber ${ }^{3}$ and Carlos Cerrada ${ }^{1}$ \\ ${ }^{1}$ Escuela Técnica Superior de Ingeniería Informática, UNED, Madrid, Spain \\ ${ }^{2} 3$ Visual Computing \& Robotics Lab, Universidad de Castilla-La Mancha, Ciudad Real, Spain \\ ${ }^{3}$ The Robotics Institute, Carnegie Mellon University, Pittsburgh, USA \\ * Corresponding author (evalero@issi.uned.es)
}

\begin{abstract}
Laser scanner data is increasingly being used for the detailed reverse engineering of buildings. This process is currently primarily manual, but recent research has shown that basic structures, such as walls, ceilings, floors, doorways, and windows, can be detected and modeled automatically. Building on this previous research, we focus on the modeling of those linear moldings that typically surround doorways, windows, and divide ceilings from walls and walls from floors. These structures may be secondary and merely ornamental, but many projects nevertheless require that they be modeled. Moldings can be difficult to model manually owing to missing data caused by occlusions or to the ambiguity caused by low data density.

Our molding modeling approach consists of two steps: 1) estimating the path of the molding; and 2) estimating the shape of the molding profile. In the first step, we iteratively update the molding's line of extrusion by optimizing the similarity of cross-sections sampled along the path, thereby compensating for imperfections in the initial orientation estimate. In the second step, a unified profile is extracted using data from the entire length of the molding, which allows for partial missing data from occlusions. The profile is then characterized by a specific shape descriptor. Finally, a KNN algorithm classifies the molding into a database which has been constructed with profiles originating from various molding manufacturers. We demonstrate the method using real 3D laser scanner data of various types of moldings, both simple and complex.
\end{abstract}

Keywords: BIM, moldings, laser scanner data, segmentation, modeling

\section{INTRODUCTION}

Over the last few decades, 3D data recording technologies have been extensively applied in the areas of civil engineering and architecture. Laser sensors have become more accurate and adaptable to be used in creating Buildings Information Models (BIM). These models are usually constructed manually, which implies intensive and complex work.

Many researchers who deal with building data processing identify semantic features, such as walls, windows, or doors in an automatic manner. Stamos [10] presents an approach which models the large planar areas of a facility with primitives and the non-planar areas with meshes via the implementation of the ball pivoting algorithm. In [9], knowledge concerning the features' sizes, positions, and topology is introduced to recognize elements in a point cloud. On another front, Vosselman et al. [11] review several techniques to extract different parameterized shapes, such as planes, cylinders and spheres by means of noninterative methods to detect clusters. Pu et al. [8] suggest a plane surface growing algorithm to distinguish between doors, windows, roof and walls. Other strategies segment planes using the RANSAC algorithm [2].

In addition to constructive elements, rooms also contain 
furniture, shelves and other objects, which create occlusions. These elements influence the detection of walls and moldings, and, before carrying out the structural element recognition process, it is therefore necessary to both detect and reconstruct the holes caused by occlusions. Adan and Huber [1] present a solution based on a labeling strategy in different data representation spaces, in which the walls are reconstructed. Castellani et al. [3] work on the reconstruction of the corners and edges of partially occluded furniture pieces in an indoor scene. Dell'acqua and Fisher [6] show a technique with which to reconstruct the shape of planar surfaces behind arbitrary occluding surfaces.

Moldings suit an emergent purpose in BIM, in either heritage or civil engineering. Few works dealing with this can be found in literature to date. In [4], several architectural rules are presented to identify features and produce a template shape library dedicated to building surveying, in which each molding is described semantically. Fuchs [7] uses 3D data originating from point clouds to architectural moldings and completes their CAD models of archeological buildings. In [4], the author proposes a method in which architectural parametric elements are "semi-automatically" adjusted to a point cloud or a photograph.

Although automatic processes are very common in geometrical shape detection, the lack of symmetry in moldings and the complexity of their geometry entail a manual molding segmentation process. In this paper, we present a solution to the automation of this identification process by segmenting windows and door frames.

The main objective of our work is to identify molding structural components. We then compare those moldings originating from the scanned room with a database created from various catalogues provided by different manufacturers.

The paper is organized as follows. Section 2 deals with the problem of molding segmentation. In Section 3, we show how to compare moldings through the calculation of certain descriptors. Section 4 presents our experimental results, and Section 5 summarizes the paper's principal contributions, along with improvements that we hope to make in the future.

\section{MOLDING SEGMENTATION}

As mentioned previously, door and window frames adhere to the walls. Various published methods [1] exist which recognize essential parts of walls, like doorways or windows. Once we know the position of these elements, it is possible to analyze their environment in order to segment, recognize and classify the moldings which are situated around them. Finally, the moldings can be added to the BIM model of the scene. This segmentation process is carried out in two steps, the first of which consists of analysing the molding alignment along its longitudinal axis, and the second characterizes the molding profile by means of a suitable descriptor. Once these two phases have been completed, we can compare the descriptor obtained with others and classify the molding into a database.

\subsection{Determining the Molding's Axis}

Doors and windows are usually rectangular in shape, signifying that their sides are parallel or perpendicular to the floor. We can easily find the approximate axis of the molding, for example, by assuming that it is horizontal or vertical or that it follows the boundary of an opening. However, real world boundaries are usually not exactly vertical or horizontal, and so it is necessary to determine the exact axis of the molding by fitting. In these circumstances, it would be necessary to study the alignment of the moldings detected in the scene.

In order to study the aforementioned alignment it is first necessary to define a region of interest (ROI). In this case, we focus our attention on an interior wall by extracting data from around a molding. Figure 1 shows an example in 
which the sensed points corresponding to a wall have been used to generate a model. The ROI, in which a molding of a window is included, is displayed.

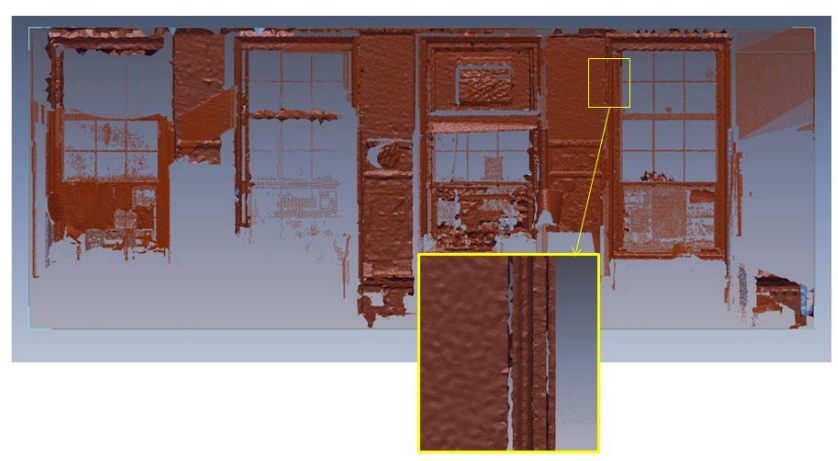

Fig. 1 Wall under review where ROI is closed.

The next step consists of dividing the ROI into various slices which are perpendicular to the longitudinal axis of the door or window. We determine the alignment of the molding by studying the data contained in these slices. Figure 2 shows a slice in which the points corresponding to the wall and the molding are displayed.
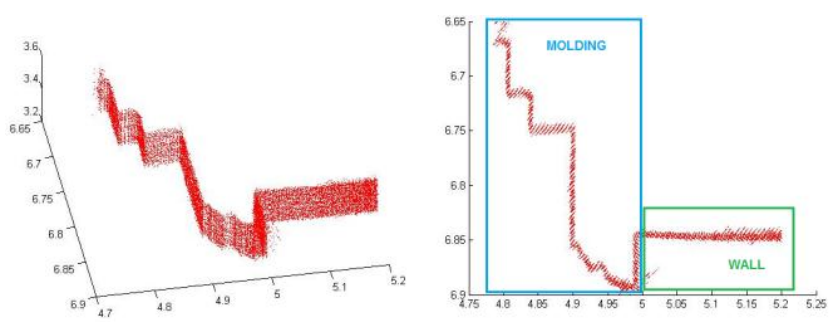

Fig. 2 Different views of the plane corresponding to a slice of the ROI.

A two-dimensional histogram of the density of points from a top view is generated. This histogram can be interpreted as an image in which the gray level indicates the number of points projected in the real area demarcated by the pixel. Then, we calculate the correlation between pairs of $2 \mathrm{D}$ histograms.

In the alignment process, two images from adjacent slices are studied and the translation for a maximum correlation $(\delta x, \delta y)$ is determined. Thus, for $k$ slices in the ROI, we obtain $k$ corresponding points $(\delta x, \delta y), i=1,2, \ldots, k$. The axis of the molding is finally achieved by adjusting all the corresponding points to a straight line (see Figure 3).

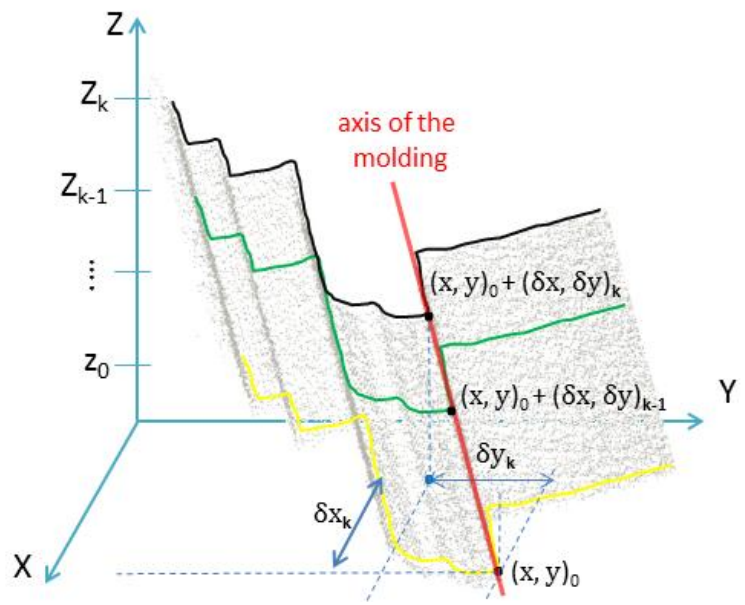

Fig. 3 Study of maximum correlation to obtain the axis of the molding.

\subsection{Molding Segmentation}

The molding descriptor is defined through an image obtained from the 2D histogram. The 2D histogram corresponding to a slice of the molding is converted into a weighted histogram in which the presence of a point has an effect on the four adjacent pixels in the image. The influence of each $3 \mathrm{D}$ point on the frequency of each pixel is inversely proportional to the distance between the point and the center of each pixel.

Once the weighted 2D histogram has been obtained, we generate an image $I$ which is composed of the most voted zones in the histogram. Figures 4 a) and b) show the weighted histogram and the function $f(x)$ obtained. A Hough Transform is calculated to determine the straight segments in the image $I$, and a piecewise function $f(x)$ is estimated to achieve the profile of the molding.

The next objective is to separate the different parts corresponding to the wall and moldings in the image $I$. The longest horizontal segment $s_{\text {wall }}$, located at one of the ends of the ROI allows us to calculate the coordinate $Y$ of the wall, $y_{\text {wall }}$. The step function $h(x)$, which fits the wall better, is determined by using the straight lines detected in the molding profile image. Thus:

$$
h[x]=\left\{\begin{array}{l}
y_{\text {wall }} \text { if } x \in(b, c) \\
0 \text { otherwise }
\end{array}\right.
$$


in which

$b=\min _{x}(\Omega), \quad c=\max _{x}(\Omega)$ and $\Omega=p(x, y) \in s_{\text {wall }}$.

The regions corresponding to the molding are segmented in image $I$ by means of the functions $f(x)$ and $h(x)$. A new binary image $S$ (see Figure 4d) is then generated, in which the points of the molding are identified. This image $S$ can be expressed as:

$$
S(x, y)=\left\{\begin{array}{l}
1 \text { if } h(x) \leq y \leq f(x) \\
0 \text { otherwise }
\end{array}\right.
$$
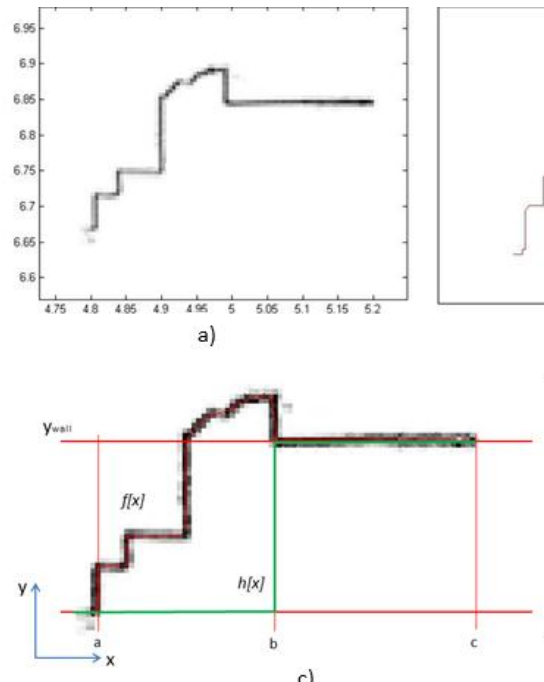

c)

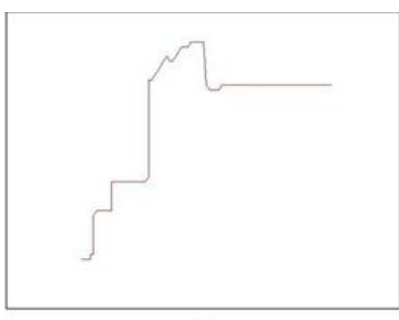

b)

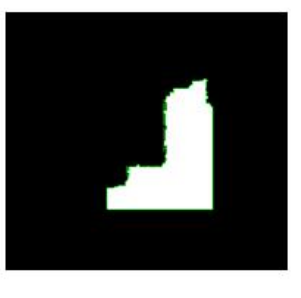

d)
Fig. 4 a) Weighted 2D histogram. b) Image $I$ and profile line $f(x)$ c) Definition of the function $h(x)$ d) Image $S$ in which molding regions are segmented.

\section{MOLDING DESCRIPTOR}

Once all the regions have been segmented, the different profiles extracted from the image $S$ are compared with a collection of models which are stored in a database. We can thus recognize the profile present in the scene, and can additionally complete some door or window frames which are incomplete owing to a lack of information in the scan data.

A descriptor for each segmented region is defined from the image $S$. The proposed descriptor consists of a normalized signature of the boundary of each region. The centroid of the profile is calculated previously in order to establish a reference point for each region.
The two parameters that define the profile descriptor are distance $(d)$ and angle $(\theta)$ from the centroid to the molding silhouette. Thus, the descriptor represents the outline of the profile in polar coordinates.

In order to avoid certain scale problems, we normalize the parameter $d$ by dividing each value of this variable by the maximum value. All the normalized distance values therefore remain in the $[0,1]$ interval regardless of the profile size. Figure 5 shows the descriptor for one of the molding regions considered.

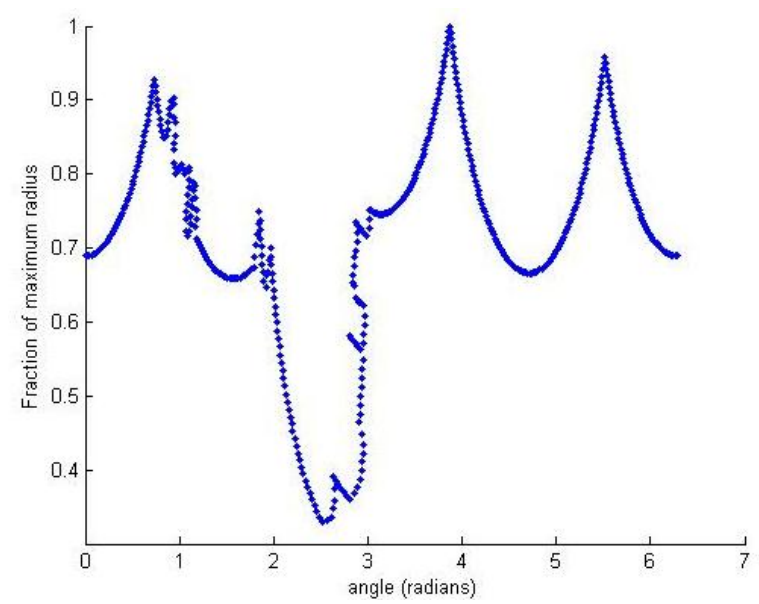

Fig. 5 Descriptor of a molding profile.

\section{EXPERIMENTAL TESTS}

The molding recognition method presented in this paper has been implemented and tested on $3 \mathrm{D}$ data provided by a laser scanner.

In spite of defects in manufacturing, occlusions in the scene, or some imprecisions in the data registration stage, our method permits the identification of the moldings which have been previously segmented from the point cloud.

The profile classification has been carried out using the KNN algorithm by evaluating the 8 nearest neighbors of the shape being tested.

Upon calculating the area contained between the two descriptors which belong to the scanned profile and to the profile stored in the database, we can determine that the similarity measure between both moldings is similar. We propose a variation of the Riemann sum to carry out this calculation. Thus, 


$$
R=\sum_{i=1}^{n}\left|d_{i}-d^{\prime}{ }_{i}\right|\left(\theta_{i}-\theta_{i-1}\right)
$$

Where $\left(\theta_{i}, d_{i}\right)$ are the pairs of points used to normalize every outline, $d_{i}$ and $d^{\prime}{ }_{i}$ being the parameterized distances for the query and the associated descriptor in the database.

A database in CAD format is provided by molding manufacturers, and a classification using a supervised learning method is carried out.

In the supervised learning process we have handled a database containing 20 profiles. The learning set has been generated by a noise injection from 0 to $10 \%$ in the original molding models. 200 profiles were used to carry out this experiment. Of these, 140 were used in the training process and 60 were used in the testing process.

We have observed that if noise is under $4 \%$ of the size of the object being recognized, then the success rate is very high $(95 \%)$, whereas if noise is over $4 \%$, the error rate is notable $(>20 \%)$. Figure 6 shows several profiles from our database that may cause greater confusion owing to their similarity.
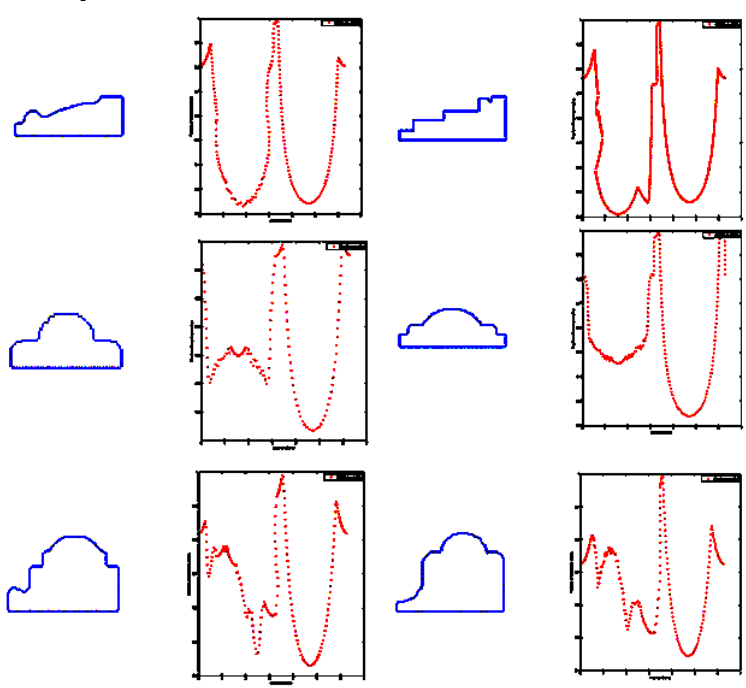

Fig. 6 Pairs of similar profiles and their shape descriptors.

A classification test was carried out by using real data from a laser scanner of ten moldings of doors and windows. The procedures described in this paper have been followed to obtain the profile of each molding and the classification process has then been carried out. Figure 7 shows the molding descriptors of a query molding (in blue) and the assigned prototype (in red).

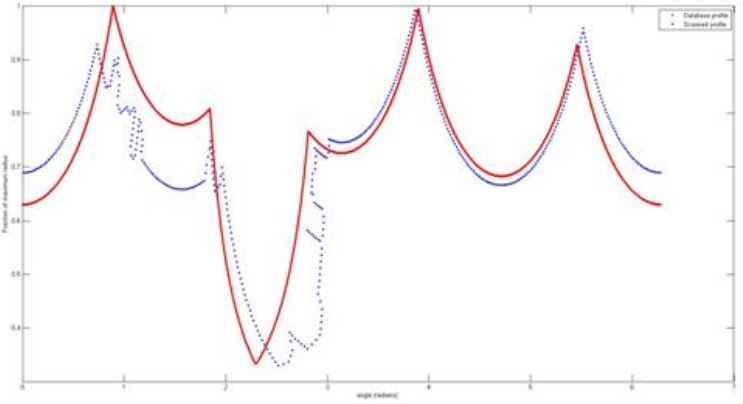

a)

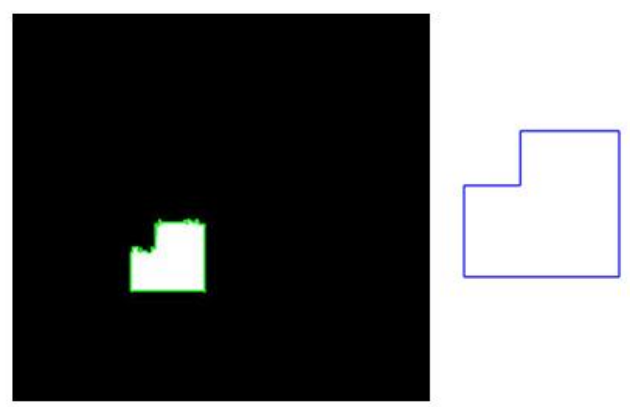

b)

Fig. 7 Classification of a molding extracted from the point cloud. a) Shape descriptors of the molding extracted from the point cloud and the matched prototype b) Images showing the molding and assigned prototype regions.

Figure 8 presents several moldings examples and the recognized $\mathrm{CAD}$ molding superimposed on the $3 \mathrm{D}$ data.
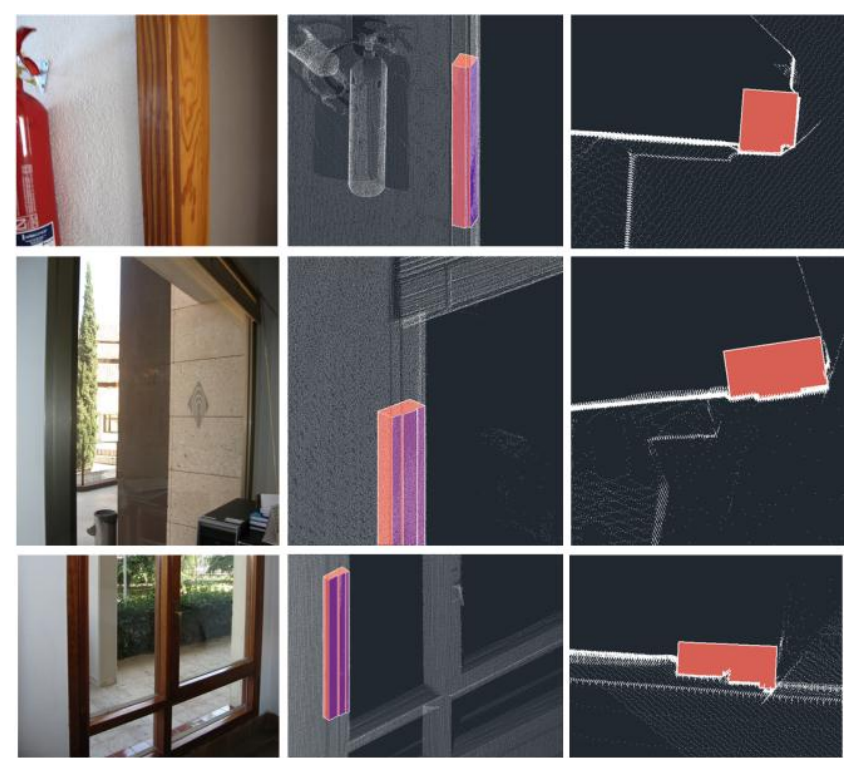

Fig. 8 Different doors and windows whose moldings have been reconstructed. 


\section{CONCLUSIONS AND FUTURE WORK}

This paper presents a proposal in the field of molding recognition from scanned data in interiors. More specifically, the work describes the procedures implemented to deal with this problem from the initial 3D data segmentation phase to the final molding classification stage.

The molding segmentation process is carried out after determining the molding axes. The regions corresponding to the moldings are then extracted with an innovative method based on the use of weighted 2D histograms obtained from a zenithal view of a wall.

With regard to the molding classification, the paper first describes how moldings can be characterized by means of a shape descriptor. A classification test based on a learning supervised KNN algorithm is then developed. Experimental results with up to 200 different profiles have been included in the work, showing the robustness of the overall method. This has led us to contemplate the future use of this technique in automatic solutions to generate BIM models, and we have high expectations as regards the success of this venture.

\section{Acknowledgment}

This research has been carried out under contract with the Spanish CICYT through the DPI-2008-05444 and DPI200914024-C02 projects. It also belongs to the activities carried out in the frame of the RoboCity2030-II excellence research network of the CAM (ref. S2009/DPI-1559). This material is based upon work supported, in part, by the National Science Foundation under Grant No. 0856558. Any opinions, findings, and conclusions or recommendations expressed in this material are those of the authors and do not necessarily reflect the views of the National Science Foundation.

\section{REFERENCES}

[1] Adan, A. and Huber, D., "3D Reconstruction of Interior Wall Surfaces Under Occlusion and Clutter". in In Proceedings of $3 D$ Imaging, Modeling, Processing,
Visualization and Transmission (3DIMPVT), Hangzhou, China, 2011 (in press).

[2] Boulaassal, H. et al. "Automatic Segmentation of Building Façades Using Terrestrial Laser Data”, Laser 07, p.65.

[3] Castellani, U. et al., "Improving environment modelling by edge occlusion surface completion", International Symposium on 3D Data Processing Visualization and Transmission, 2002.

[4] Chevrier, C. and Perrin, J.P. "Laser Range Data, Photographs and Architectural Components", ISPRS, 2008.

[5] De Luca, L. et al., "A generic formalism for the semantic modeling and representation of architectural elements", Vis. Comput., 23:181--205, 2007.

[6] Dell'acqua, F. and Fisher, R., "Reconstruction of Planar Surfaces Behind Occlusions in Range Images",IEEE Trans. on Pattern Analysis and Machine Intelligence, 24, 2002.

[7] Fuchs, A., "Outils numeriques pour le relevé architectural et la restitution archéologique", $\mathrm{PhD}$ thesis, Université Henri Poincaré, Nancy 1, 2006.

[8] Pu, S. and Vosselman, G., "Automatic extraction of building features from terrestrial laser scanning", IEVM06, 2006.

[9] Pu, S. and Vosselman, G., "Knowledge based reconstruction of building models from terrestrial laser scanning data", ISPRS Journal of Photogrammetry and Remote Sensing, 64(6):575 - 584, 2009.

[10] Stamos, I. et al., "3D Modeling Using Planar Segments and Mesh Elements", 3D Data Processing, Visualization, and Transmission, Third International Symposium, pages 599 -606, 2006.

[11] Vosselman, G. et al., "Recognising Structure in Laser Scanner Point Clouds". International Archives of Photogrammetry, Remote Sensing and Spatial Information Sciences, pages 33-38, 2004. 